\title{
Surface Charge Modulation and Reduction of Non-Linear Electrolytic Screening in FET-Based Biosensing
}

\author{
Mihir Gupta ${ }^{\circledR}$, Member, IEEE, Sybren Santermans $^{\circledR}$, Member, IEEE, \\ Geert Hellings ${ }^{\circledR}$, Senior Member, IEEE, Liesbet Lagae, \\ Koen Martens, Member, IEEE, and Willem Van Roy ${ }^{(1)}$
}

\begin{abstract}
We experimentally investigate the influence of non-linear electrolytic screening by the electric double layer (EDL) and its impact on the field-effect transistor (FET) sensitivity to charged (bio)molecules. We use DNA hybridization to a PNA capture probe layer as model system. By co-depositing positively or negatively charged blocker molecules together with the PNA capture probes on the negatively charged $\mathrm{SiO}_{2}$ surface of the FET, we control the overall surface charge and modulate the strength of local ion concentration within the EDL. We observe a FET signal enhancement of up to $51 \%$ when the overall charge including the captured analyte itself swings roughly symmetrically around zero during capture, as confirmed by zeta potential measurements. Surface plas-

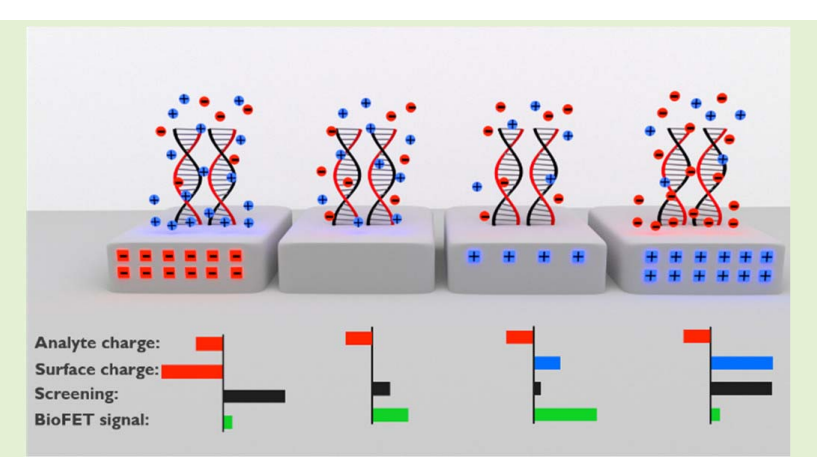
mon resonance measurements rule out a change in captured analyte density as the origin of the enhancement in sensitivity. This confirms that excess screening caused by the large local ion concentration, which increases non-linearly with potential in the EDL, is responsible for a loss in sensitivity in bioFETs with a charged surface. These experimental findings agree with the early theoretical works that find a low surface potential to be desirable for the best bioFET performance.
\end{abstract}

Index Terms-Biosensing, pH sensing, field-effect transistor, electrolytic screening, surface charge, DNA hybridization, electric double layer.

\section{INTRODUCTION}

$\mathbf{I}$ T IS well established that electrostatic screening of biomolecular charge by electrolyte ions significantly impacts potentiometric biosensing using field-effect transistors (FETs). The screening increases with increase in ionic strength and consequently FET-based sensing is preferentially done in low ionic strength buffers [1]-[3]. However, much less attention has been given to the phenomenon of increased non-linear

Manuscript received July 12, 2020; accepted October 11, 2020. Date of publication October 15, 2020; date of current version January 15 2021. The associate editor coordinating the review of this article and approving it for publication was Dr. Irene Taurino. (Corresponding author: Mihir Gupta.)

Mihir Gupta and Liesbet Lagae are with imec, 3001 Leuven, Belgium, and also with the Department of Physics and Astronomy (Physics of Soft Matter and Biophysics), Katholieke Universiteit Leuven, 3000 Leuven, Belgium (e-mail: mihir.gupta @ imec.be).

Sybren Santermans is with imec, 3001 Leuven, Belgium, and also with the Department of Materials Science [Functional materials (SIEM)], Katholieke Universiteit Leuven, 3000 Leuven, Belgium.

Geert Hellings and Willem Van Roy are with imec, 3001 Leuven, Belgium (e-mail: vanroy@imec.be).

Koen Martens is with imec, 3001 Leuven, Belgium, and also with the Department of Physics and Astronomy (Semiconductor Physics), Katholieke Universiteit Leuven, 3000 Leuven, Belgium (e-mail: koen.martens@imec.be).

Digital Object Identifier 10.1109/JSEN.2020.3031469 electrolytic screening when an electric double layer (EDL) is present at the sensor surface, e.g. in response to surface charges. The local ion concentration increases exponentially with the local potential (Boltzmann distribution), and strongly exceeds the bulk ionic strength as soon as the local potential exceeds a few times $k_{B} T / q(=25 \mathrm{mV})$. This results in a strong and non-linear increase in electrolytic screening.

Landheer et al. [4], [5] identified this phenomenon in a 1-D numerical model for planar devices where the biomolecular layer was modeled as an ion-permeable 'membrane' of finite thickness, separated from the surface by a thin Stern layer. Later, Wunderlich et al. [6] and Landheer et al. [7] presented analytical solutions for the simplified cases where the analytes are positioned directly at the oxide-electrolyte interface, i.e. in a plane with zero thickness or where the biolayer has a finite thickness, respectively. This resulted in a much more accessible analytical treatment, and the simple dependency between biomolecular sensitivity and the pre-exiting surface potential for the charge plane [6],

$$
\frac{d \Psi_{0}}{d \sigma_{\text {ana }}} \sim \frac{1}{\cosh \left(\frac{z q \Psi_{0}}{2 k_{B} T}\right)}
$$


with $\Psi_{0}$ the potential at the oxide-electrolyte interface with respect to the bulk electrolyte potential, $\sigma_{\text {ana }}$ the analyte charge density (located in the interfacial plane), and $\mathrm{z}$ the valency of the bulk electrolyte $(\mathrm{z}=1$ for $\mathrm{NaCl})$. Note that the pre-existing EDL potential can not only result from fixed interface or oxide charges, but also from analyte charges already present on the surface. In other words, the differential sensitivity also tends to go down as more analyte charges are captured. A few other groups also noted this non-linear screening effect [8] and have explored it using numerical analysis [9]. More recently, Shoorideh and Chui [10] modelled the independent co-optimization of the EDL (low potential drop i.e. low integrated charge density) and of the FET (e.g. low channel charge density in the subthreshold regime) using both a front and back gate, and they suggested a practical way to fine-tune the device by engineering the amount of surface charge near the electrolyte/oxide interface. However, there has been very limited follow-up [11] and to the best of our knowledge, no detailed experimental verification of this screening effect has been reported.

In this paper, we provide the experimental demonstration of the non-linear screening phenomenon and we show that an improved FET-based biomolecular sensitivity can be obtained by optimizing the sensor surface charge with charged blocker molecules. For this, we used a peptide nucleic acid (PNA) - deoxyribonucleic acid (DNA) hybridization system as a model in combination with FETs fabricated completely using CMOS technology. In order to modify the surface charge, we co-deposited positively charged $\left(-\mathrm{NH}_{2}\right.$ terminated) or negatively charged (-COOH terminated) blocker molecules together with the PNA capture probes. This was followed by a noncomplementary DNA (ncDNA) incubation step (as negative control) and a complementary DNA (cDNA) hybridization step. By varying the surface blocker density for positive and negative polarities we were able to demonstrate the impact of non-linear EDL screening on hybridized cDNA FET signal transduction by controlling the surface charge. Streaming potential measurements were used to determine the zeta potential after various surface modifications. Surface plasmon resonance measurements were used to check for any secondary influence of the blockers on the degree of cDNA hybridization.

\section{Materials and Methods}

\section{A. Reagents}

To prepare $150 \mathrm{mM}$ and $1.5 \mathrm{mM}$ buffers, phosphate buffered saline (PBS) 10X solution (1.5 M) (by InvitrogenTM, purchased from ThermoFisher Scientific, catalog \#AM9624) was diluted with ultrapure de-ionized water (DIW). PNA with dibenzocyclooctyne (DBCO) modification purified with highperformance liquid chromatography (HPLC) (DBCO-C6-N term/AAC TCT CCA CTC ACC/C-term), was purchased from Panagene Inc and was suspended in DIW to a concentration of $50 \mu \mathrm{M}$, aliquoted and stored at $-20{ }^{\circ} \mathrm{C}$. Complementary single stranded DNA (5/GGT GAG TGG AGA GTT/3) and non-complementary single stranded DNA (5/AAC ACA CAA CAC ACC/3) purified with HPLC (for hybridization and for negative control experiments respectively) were purchased from Integrated DNA Technologies BVBA (IDT). The
DNA was first dissolved at a concentration of $100 \mu \mathrm{M}$ in a buffer (pH 8) containing $10 \mathrm{mM}$ tris (product \#T1503, Sigma-Aldrich) and $0.1 \mathrm{mM}$ ethylenediaminetetraacetic acid (EDTA) (product \#431788, Sigma-Aldrich), aliquoted and then stored at $-20{ }^{\circ} \mathrm{C}$. Isopropyl alcohol (IPA) was purchased from KMG chemicals (product \#135825). The $\mathrm{pH}$ adjustments for the buffers were done using $7 \% \mathrm{HCl}$ solution (product \#130471, KMG chemicals) or $2 \mathrm{M} \mathrm{NaOH}$ solution (pellets purchased from Supelco, product \# 106469). The molecule 11-azidoundecyltrimethoxysilane $\left(\mathrm{N}_{3}\right.$-silane) (95\% pure, CAS: 334521-23-2, article no. AB258623) for surface functionalization was purchased from abcr $\mathrm{GmbH}$, Germany. The DBCO modified charged blocker molecules (with polyethylene glycol (PEG) linker), DBCO-PEG4-NH2 (CAS: 1255942-08-5) and DBCO-PEG4-COOH (CAS: 1537170-85-6) with HPLC purification ( $>95 \%$ purity) were purchased from Jena Bioscience GMBH, Germany. These blocker molecules were first dissolved in dimethyl sulfoxide (DMSO) to a final concentration of $50 \mathrm{mM}$, aliquoted and then stored at $-20^{\circ} \mathrm{C}$.

\section{B. Device Fabrication}

The FETs in this work were inversion-mode p-channel devices fabricated completely in a CMOS pilot line [12], [13]. They were fabricated on $300 \mathrm{~mm}$ silicon-on-insulator (SOI) wafers (supplied by Soitec) with a $145 \mathrm{~nm}$ thick buried oxide (BOX) and $88 \mathrm{~nm}$ thick silicon device layer (resistivity $\sim 12 \Omega$ $\mathrm{cm})$. First, the Si device layer thickness was reduced to $26 \mathrm{~nm}$ by successive oxidation and wet etching. Fins with widths from $1000 \mathrm{~nm}$ to $60 \mathrm{~nm}$ and lengths from $3000 \mathrm{~nm}$ to $450 \mathrm{~nm}$ were defined using an advanced patterning approach based on $193 \mathrm{~nm}$ immersion lithography and hard mask shrinking [14], [15]. The channel doping is set at $2 \times 10^{17} \mathrm{~cm}^{-3}$ (phosphorous) and the source/drain regions received high contact implant doping (>1 $\times 10^{20} \mathrm{~cm}^{-3}$, boron). This was followed by the growth of a $4 \mathrm{~nm}$ thick thermal $\mathrm{SiO}_{2}$ layer as gate oxide. Next, CVD polysilicon was deposited to act as sacrificial layer for final cavity opening for electrolyte access to the $\mathrm{Si}$ fin. This was followed by gate patterning and an anneal at $1030{ }^{\circ} \mathrm{C}$.

Next, an oxide passivation layer was deposited, followed by opening of the source and drain contact, routing-out the connections towards the bond pads and the final oxide passivation layer deposition. The FET cavity and the bondpads were opened using a combination of dry and wet etch processes to expose the FET gate oxide and the bondpad metal.

\section{Surface Chemistry}

A self-assembled monolayer (SAM) of $\mathrm{N}_{3}$-silane was deposited on the sensor surface using chemical vapor deposition [16]. This was done in a vacuum chamber by vaporizing $120 \mu \mathrm{l}$ of $\mathrm{N}_{3}$-silane at $145{ }^{\circ} \mathrm{C}$ and at $25 \mathrm{mbar}$ pressure for 2 hours, during which a uniform monolayer of the molecule was deposited on the surface. After the deposition, the dies were first rinsed with acetone and IPA and blow dried with $\mathrm{N}_{2}$. The DBCO conjugated PNA with/without blocker molecules were immobilized on the surface by reacting with the azide terminal of the SAM.

\section{Biacore Setup and Protocol}

Surface plasmon resonance (SPR) experiments were performed on a Biacore 3000 system. The substrates were made 


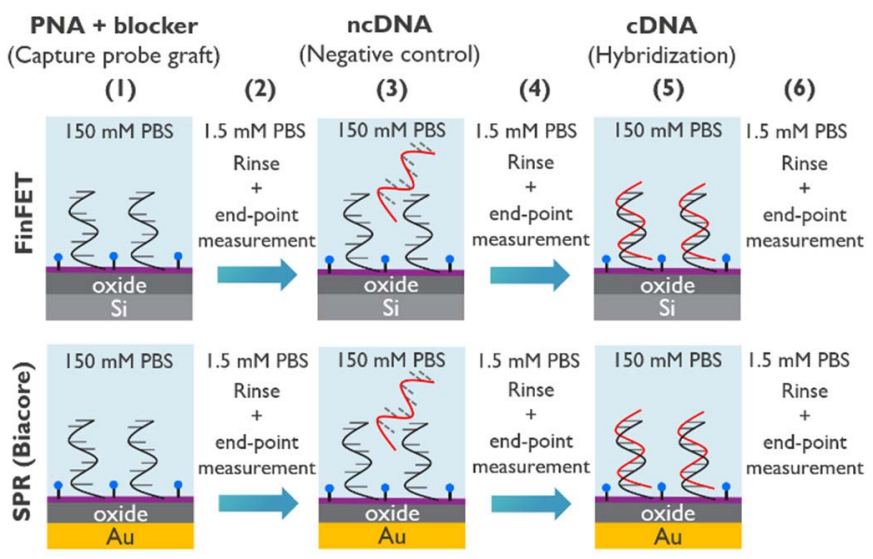

Fig. 1. PNA (+ blocker), ncDNA and cDNA immobilization protocol for the reference SPR based Biacore 3000 measurements (on oxide covered Au surface [17]) and for the FET electrical sensing measurements (corresponding to protocol in Table I); the purple layer represents $\mathrm{N}_{3}$-silane SAM. (1) $1 \mu \mathrm{M}$ PNA (shown as black single strands) + variable blocker concentration (shown as blue circular dots) in $150 \mathrm{mM}$ PBS for 30 minutes, (3) $1 \mu \mathrm{M}$ ncDNA in $150 \mathrm{mM}$ PBS for 15 minutes as negative control and should not bind to the PNA probe, (5) $1 \mu \mathrm{M} \mathrm{cDNA}$ in $150 \mathrm{mM}$ PBS for 15 minutes to obtain (saturated) hybridization with PNA probe. Each incubation step was followed by (2), (4) and (6) thorough rinsing and end-point measurements using 1.5 mM PBS for both FET as well as SPR. This ensured similar incubation as well measurement conditions for FETs and Biacore 3000 reference measurements [13], [17].

from $300 \mu \mathrm{m}$ thick, $1 \times 1 \mathrm{~cm}^{2}$ borosilicate glass (D 263®T eco Thin Glass, purchased from Schott AG), coated with a stack of $2 \mathrm{~nm} \mathrm{Ti}, 40 \mathrm{~nm} \mathrm{Au}$ (evaporated), $2 \mathrm{~nm} \mathrm{Ti}$ and $10 \mathrm{~nm} \mathrm{SiO} 2$ (sputtered) [17]. The samples were cleaned with acetone and IPA, followed by a 15 min $\mathrm{UV}_{-} \mathrm{O}_{3}$ treatment $(28$ $32 \mathrm{~mW} / \mathrm{cm}^{2}$ at $253.7 \mathrm{~nm}$ ) prior to $\mathrm{N}_{3}$-silane $\mathrm{SAM}$ deposition.

The sample injections (PNA/blocker/DNA) were done in $150 \mathrm{mM}$ PBS, while for running buffer (which flows between each successive sample injection) $1.5 \mathrm{mM}$ PBS was used. To quantify the amount of PNA/blocker/DNA immobilized on the surface, end-point measurements were done in $1.5 \mathrm{mM}$ PBS running buffer (before and after each sample injection step). This was done to replicate similar conditions as in the end-point FET electrical measurements [13], where $150 \mathrm{mM}$ PBS was used during PNA/blocker/DNA incubation steps (when no FET electrical measurements were being performed) and $1.5 \mathrm{mM}$ PBS was used during FET electrical measurements (after each PNA/blocker/DNA incubation step) as shown in Figure 1. The flow rate for Biacore 3000 was set at $5 \mu \mathrm{l} / \mathrm{min}$. First, $150 \mu \mathrm{l}$ (30 mins) of $1 \mu \mathrm{M}$ PNA along with blocker molecule (concentration varied) in $150 \mathrm{mM}$ PBS buffer was injected into the channel. This was followed by a $75 \mu 1(15 \mathrm{~min})$ injection of $1 \mu \mathrm{M}$ non-complementary DNA (ncDNA) and then a $75 \mu \mathrm{l}(15 \mathrm{~min})$ injection of $1 \mu \mathrm{M}$ complementary DNA (cDNA), both in $150 \mathrm{mM}$ PBS, which was long enough to obtain a saturated cDNA hybridization signal.

\section{E. Streaming Potential Measurements}

Zeta potential $(\zeta)$ measurements using the streaming potential technique were performed on a SurPASS ${ }^{\mathrm{TM}} 3$ system (by Anton Paar). We used planar Si samples $\left(1 \times 2 \mathrm{~cm}^{2}\right)$
TABLE I

InCUBATION STEPS AND PROTOCOL FOR FET ELECTRICAL MEASUREMENTS

\begin{tabular}{llll}
\hline$\#$ & Protocol steps & $\begin{array}{l}\text { Incubation } \\
\text { time }(\mathrm{min})\end{array}$ & $\begin{array}{l}\text { PBS buffer } \\
\text { ionic strength } \\
(\mathrm{mM})\end{array}$ \\
\hline 1 & $\begin{array}{l}\text { Capture probe + blocker grafting: } \\
1 \mu \mathrm{M} \text { PNA + (variable) blocker }(400 \\
\mu \mathrm{l}) \text { injection }\end{array}$ & 30 & 150 \\
2 & $\begin{array}{l}\text { (Rinsing + I-V measurements) } \times \mathrm{N} \\
\text { Negative control: }\end{array}$ & - & 1.5 \\
3 & $\begin{array}{l}(1 \mu \mathrm{M} \text { ncDNA }(500 \mu \mathrm{l}) \text { injection) } \\
\text { (Rinsing + I-V measurements) })\end{array}$ & 15 & 150 \\
5 & $\begin{array}{l}\text { Hybridization: } \\
(1 \mu \mathrm{M} \text { cDNA }(500 \mu \mathrm{l}) \text { injection })\end{array}$ & 15 & 1.5 \\
6 & \begin{tabular}{l} 
(Rinsing + I-V measurements $) \times \mathrm{N}$ \\
\hline
\end{tabular} & - & 150 \\
\hline
\end{tabular}

with a $100 \mathrm{~nm}$ thick thermally grown $\mathrm{SiO}_{2}$ on top. The zeta potential was measured while varying the $\mathrm{pH}$ of the $1 \mathrm{mM}$ $\mathrm{KCl}$ electrolyte using $\mathrm{HCl}$ and $\mathrm{NaOH}$. To minimize $\mathrm{pH}$ drift of the electrolyte, $\mathrm{N}_{2}$ gas was continuously bubbled through the closed beaker containing the electrolyte. The protocols for surface functionalization and PNA/blocker/DNA immobilization for these samples was same as that used for Biacore (and FET electrical measurements), i.e. $\mathrm{N}_{3}$-silane deposition followed by PNA-blocker co-deposition and DNA incubation steps in $150 \mathrm{mM}$ PBS.

\section{F. Electrical Measurement Setup}

A $60 \mu 1$ silicone flow cell (by Grace Bio-Labs, SKU: 621101-S) was mounted on the sample die and connected to a flow-through true $\mathrm{Ag} / \mathrm{AgCl}$ electrode (provided by Microelectrodes Inc. product \#16-702), which was used to apply gate-source bias $\left(\mathrm{V}_{\mathrm{GS}}\right)$ through the electrolyte [18]. The semiautomatic measurements were performed using a Keithley 2602 on a nano-probe station (by SUSS Microtech). The measurements were performed in darkness with drain-source bias $\left(\mathrm{V}_{\mathrm{DS}}\right)$ of $-50 \mathrm{mV}$ and an integration time of $40 \mathrm{~ms}$. The data reported in this paper were measured on 250 and $1000 \mathrm{~nm}$ wide and 1000 and $3000 \mathrm{~nm}$ long FETs.

\section{G. Electrical Measurement Protocol}

First, a mixture of IPA (75\%) and $1.5 \mathrm{mM}$ PBS (25\%) was flushed through the flowcell to ensure the proper wetting of the cavities. This was followed by immediate rinsing with $1.5 \mathrm{mM}$ PBS to remove of any leftover IPA in the flowcell. The subsequent steps for PNA + blocker graft and DNA hybridization are listed in Table I.

We used constant current method to quantify the shifts observed after cDNA and ncDNA incubations, where the voltage $\mathrm{V}_{0}$ was defined as the gate voltage $\left(\mathrm{V}_{\mathrm{GS}}\right)$ for which the drain-source current $\mathrm{I}_{\mathrm{DS}}=1 \mathrm{nA}$, at $\mathrm{V}_{\mathrm{DS}}=-50 \mathrm{mV}$. The transfer characteristics ( $\mathrm{I}_{\mathrm{DS}}$ vs $\mathrm{V}_{\mathrm{GS}}$ ) of the FETs were measured until $\mathrm{I}_{\mathrm{DS}}$ crossed $1 \mathrm{nA}$ and the corresponding $\mathrm{V}_{0}$ value was extracted for each measurement and for each incubation phase. Multiple rinsing (1.5 mM PBS, $500 \mu \mathrm{l})$ and 


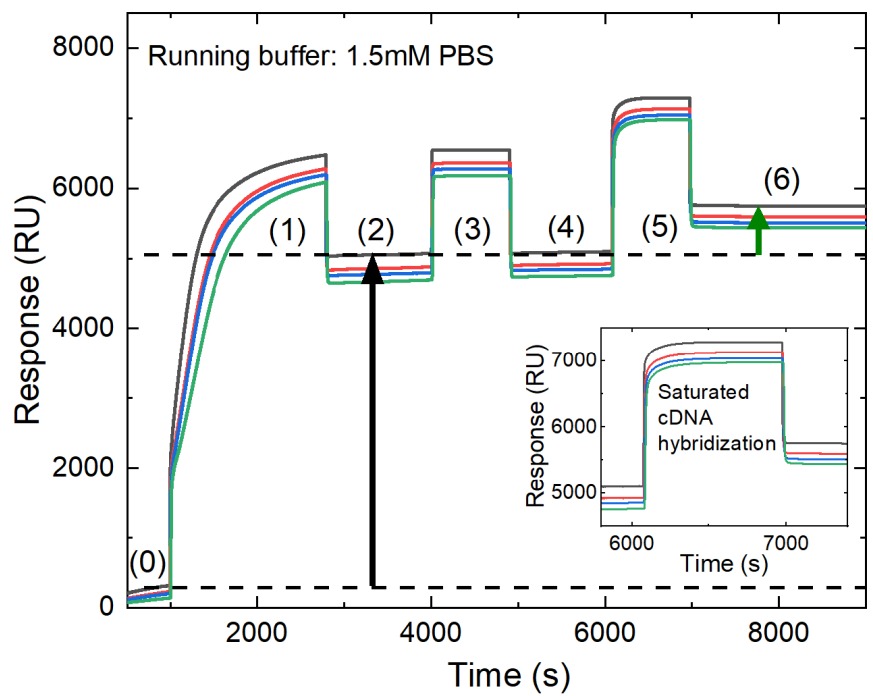

Fig. 2. PNA-DNA hybridization using Biacore 3000 , with different colours indicating the 4 different channels; (0), (2), (4), (6) $1.5 \mathrm{mM}$ PBS was used as running buffer which flows between each successive sample injection, (1) $1 \mu \mathrm{M}$ PNA $+1 \mu \mathrm{M}-\mathrm{NH}_{2}$ blocker injection in $150 \mathrm{mM}$ PBS for 30 minutes gave a response of $4607 \pm 90 \mathrm{RU}$ (black arrow), (3) $1 \mu \mathrm{M}$ ncDNA injection in $150 \mathrm{mM}$ PBS for 15 minutes gave a response of 27 $\pm 17 \mathrm{RU}$, (5) $1 \mu \mathrm{M}$ cDNA injection in $150 \mathrm{mM}$ PBS for 15 minutes reaches saturation for hybridization (as shown in the inset) and gave a response of $671 \pm 13 \mathrm{RU}$ (green arrow). The hybridized cDNA does not de-hybridize when the buffer is returned back from $150 \mathrm{mM}$ PBS to $1.5 \mathrm{mM}$ PBS as shown by a stable response in (6).

end-point electrical measurement (1.5 mM PBS) steps were performed (Steps \# 2, 4, and 6 in Table I) after each incubation step, until stable I-V characteristics were obtained. During the PNA/DNA incubation steps (\# 1, 3 and 5 in Table I), the FET device was in floating condition.

\section{Results And Discussion}

\section{A. Influence of PNA-Blocker Co-Deposition on DNA Hybridization}

To understand the impact of non-linear screening on DNA hybridization sensing, we modified the surface potential by co-immobilizing PNA probes with variable concentrations of charged blocker molecules, $\mathrm{PEG}_{4}-\mathrm{NH}_{2}\left(p K_{a, N H_{2}} \cong 9.8\right.$, positively charged at $\mathrm{pH} 7.2)$ and $\mathrm{PEG}_{4}-\mathrm{COOH}\left(p K_{a, \mathrm{COOH}} \cong\right.$ 2.4, negatively charged at $\mathrm{pH}$ 7.2) [19]. The dependence of the amount of DNA hybridization on the blocker concentration used during the grafting of the PNA capture probes (with a constant $1 \mu \mathrm{M}$ PNA) was measured using SPR (Biacore 3000 system).

Figure 2 shows a typical Biacore 3000 sensogram. The amount of immobilized PNA (+ blocker) or hybridized DNA was quantified by taking end-point measurements in running buffer i.e. the difference of the response units before and after each injection cycle, when the running buffer was flowing.

Multiple PNA-blocker co-depositions were carried out by increasing the bulk blocker concentration, while keeping a constant $1 \mu \mathrm{M}$ PNA concentration, followed by ncDNA and cDNA hybridization steps for every PNA-blocker co-deposition. The end-point saturated measurement data for all these experiments is shown in Figure 3.

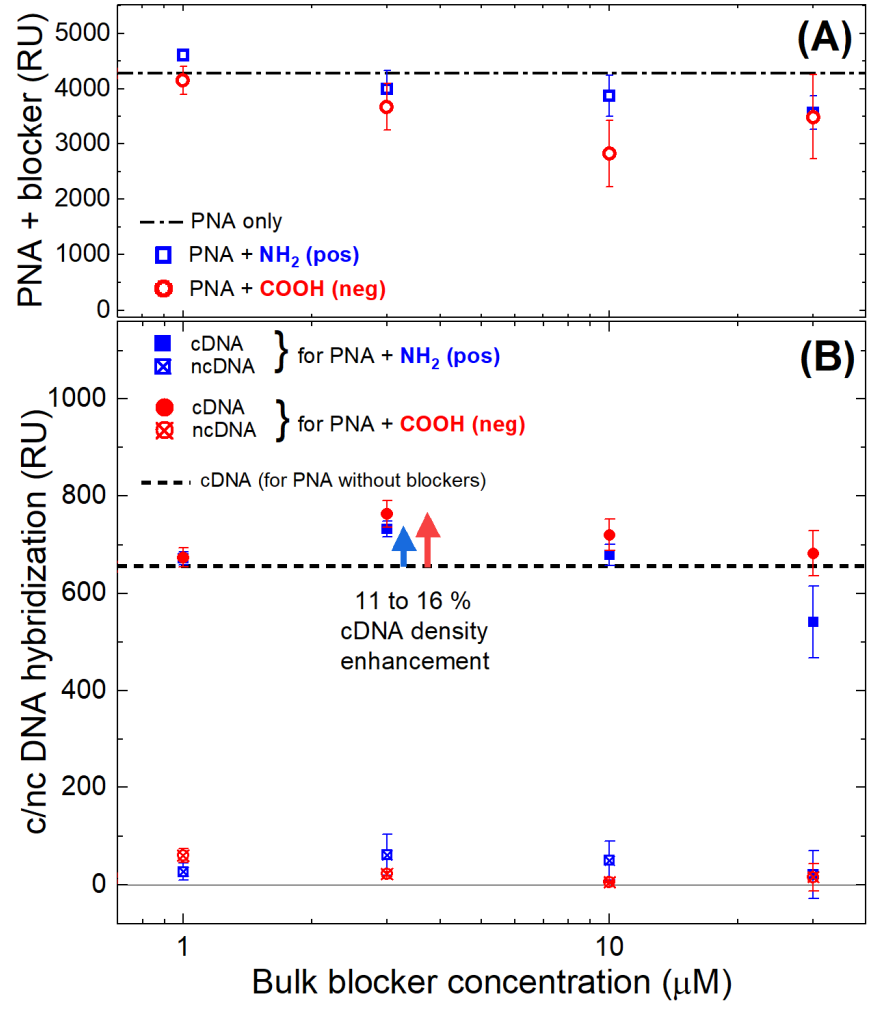

Fig. 3. (A) Total PNA + blocker signal (where 1 Biacore $R U \approx 1 \mathrm{pg} / \mathrm{mm}^{2}$ ) as function of the positive $\left(\mathrm{NH}_{2}\right)$ and negative $(\mathrm{COOH})$ bulk blocker concentrations with increase in bulk blocker concentration (molecular weight $\sim 0.5 \mathrm{kDa}$ ) co-deposited with $1 \mu \mathrm{M}$ PNA (4.2 kDa). The dashdot line represents a full PNA layer (deposited without blockers). The error bars represent the standard deviation for PNA (+blocker) signal on 4 different channels. (B) Saturated complementary (cDNA) and noncomplementary DNA (ncDNA) hybridization signals. The dashed line represents mean saturated cDNA hybridization of $656 \pm 8 \mathrm{RU}$ (equivalent to $8.3 \times 10^{4} \mathrm{cDNA} / \mu \mathrm{m}^{2}$ ) to a full PNA probe layer (without blockers).

The saturated cDNA hybridization on a full PNA layer (without blocker molecules) gave a mean response of $656 \pm 8 \mathrm{RU}$, which is approximately equivalent to $8.3 \times$ $10^{4} \mathrm{cDNA} / \mu \mathrm{m}^{2}$ (assuming 1 Biacore response unit (RU) $=$ $1 \mathrm{pg} / \mathrm{mm}^{2}$ [20], [21]), and is shown as a dashed line in Figure 3 (B). With increase in blocker content, the PNA probe density reduces somewhat (Figure $3(\mathrm{~A})$ ), causing at first an increase in PNA-DNA hybridization (Figure 3 (B)) due to the reduction of steric hindrance [22], [23]. We observed a maximum enhancement of 11 to $16 \%$ in DNA hybridization (up to $9.7 \times 10^{4} \mathrm{cDNA} / \mu \mathrm{m}^{2}$ ) at approximately $3 \mu \mathrm{M}$ blocker concentration (Figure $3(\mathrm{~B})$ ), compared to DNA hybridization on a pure PNA layer without blockers. With further increase in blocker concentration, the PNA probe density starts to decline significantly, causing a drop in DNA hybridization.

We observe no significant difference in the amount of PNA-DNA hybridization for positive versus negative blocker concentration variation. This indicates that the use of $150 \mathrm{mM}$ ionic strength buffer during the immobilization steps (PNA/blocker/DNA) screens most of the molecular and surface charge, thereby minimizing analyte (DNA) to surface and analyte electrostatic interactions. 


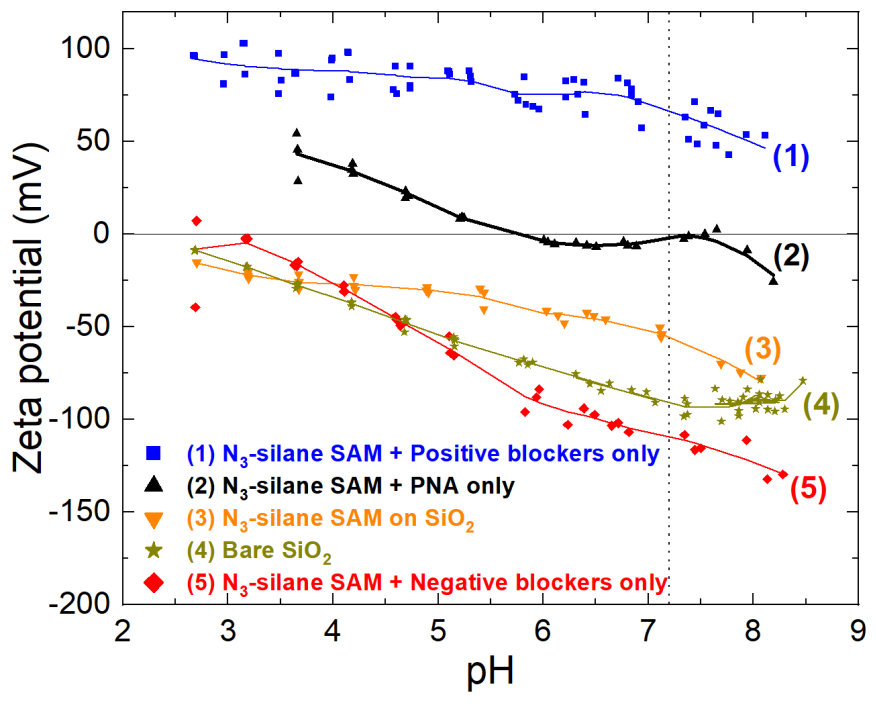

Fig. 4. Zeta potential versus $\mathrm{pH}$ of the electrolyte $(1 \mathrm{mM} \mathrm{KCl})$ for surfaces with various conditions and treatments, with a dotted vertical line at $\mathrm{pH}$ 7.2. Curves \#4 and \#3 show the zeta potentials for bare $\mathrm{SiO}_{2}$ surface $(-90 \mathrm{mV}$ at $\mathrm{pH} 7.2)$ and after $\mathrm{N}_{3}$-silane SAM deposition $(-50 \mathrm{mV}$ at $\mathrm{pH}$ 7.2) respectively. Curves \#1 and \#5 show the $\mathrm{N}_{3}$-silane SAM coated surfaces with saturated positive and negative blocker deposition (without PNA), modulating the zeta potential from $+75 \mathrm{mV}$ down to $-115 \mathrm{mV}$ at $\mathrm{pH} 7.2$ respectively. For the surface saturated only with PNA (without blockers), the zeta potential was very close to zero $\mathrm{mV}$ at $\mathrm{pH} 7.2$ (curve \#2). Markers: data points, lines: after smoothing using the locally estimated scatterplot smoothing algorithm (LOESS).

\section{B. Impact of Surface Treatments on Surface Potential}

The influence of the PNA-blocker co-depositions on the potential distribution close to the surface was evaluated using streaming potential measurements which gives access to the zeta potential $(\zeta)$. This is the potential at the slip plane, which we can assume to lie outside the dense functionalization layers, and this gives an indication of the net charge of the surface plus the functionalization layer. The bare $\mathrm{SiO}_{2}$ surface was measured to be negatively charged with the zeta potential about $-90 \mathrm{mV}$ at $\mathrm{pH} 7.2$ (Figure 4, curve \#4). After $\mathrm{N}_{3}$-silane $\mathrm{SAM}$ deposition on the $\mathrm{SiO}_{2}$ samples (and before PNA or blocker grafting), the negative surface charge reduced, and the zeta potential increased to $-50 \mathrm{mV}$ (curve \#3). By adding a saturated layer of blocker molecules (without PNA), the zeta potential could be tuned to be strongly positive (up to $+75 \mathrm{mV}$ (curve \#1)) using $-\mathrm{NH}_{2}$ terminated blocker or strongly negative $(-115 \mathrm{mV}$ (curve \#5)) using - $\mathrm{COOH}$ terminated blocker, at $\mathrm{pH}$ 7.2, as shown in Figure 4. For an $\mathrm{N}_{3}$-silane SAM coated $\mathrm{SiO}_{2}$ surface with a saturated density of only PNA molecules (without blockers), the zeta potential was very close to $0 \mathrm{mV}$ (curve \#2). These zeta potential measurements demonstrate the strong control of near-surface potentials by the selection of the appropriate type and concentration of charged blocker molecules.

\section{Impact of Non-Linear Screening on FET Sensitivity}

A typical PNA-DNA hybridization sensing experiment for a $1000 \mathrm{~nm}$ long and $1000 \mathrm{~nm}$ wide FET with its transfer curves is shown in Figure 5 (A). The gate voltage was swept down from $0 \mathrm{~V}$ until the $\mathrm{I}_{\mathrm{DS}}$ crossed $1 \mathrm{nA}$ (Figure 5 (A)).
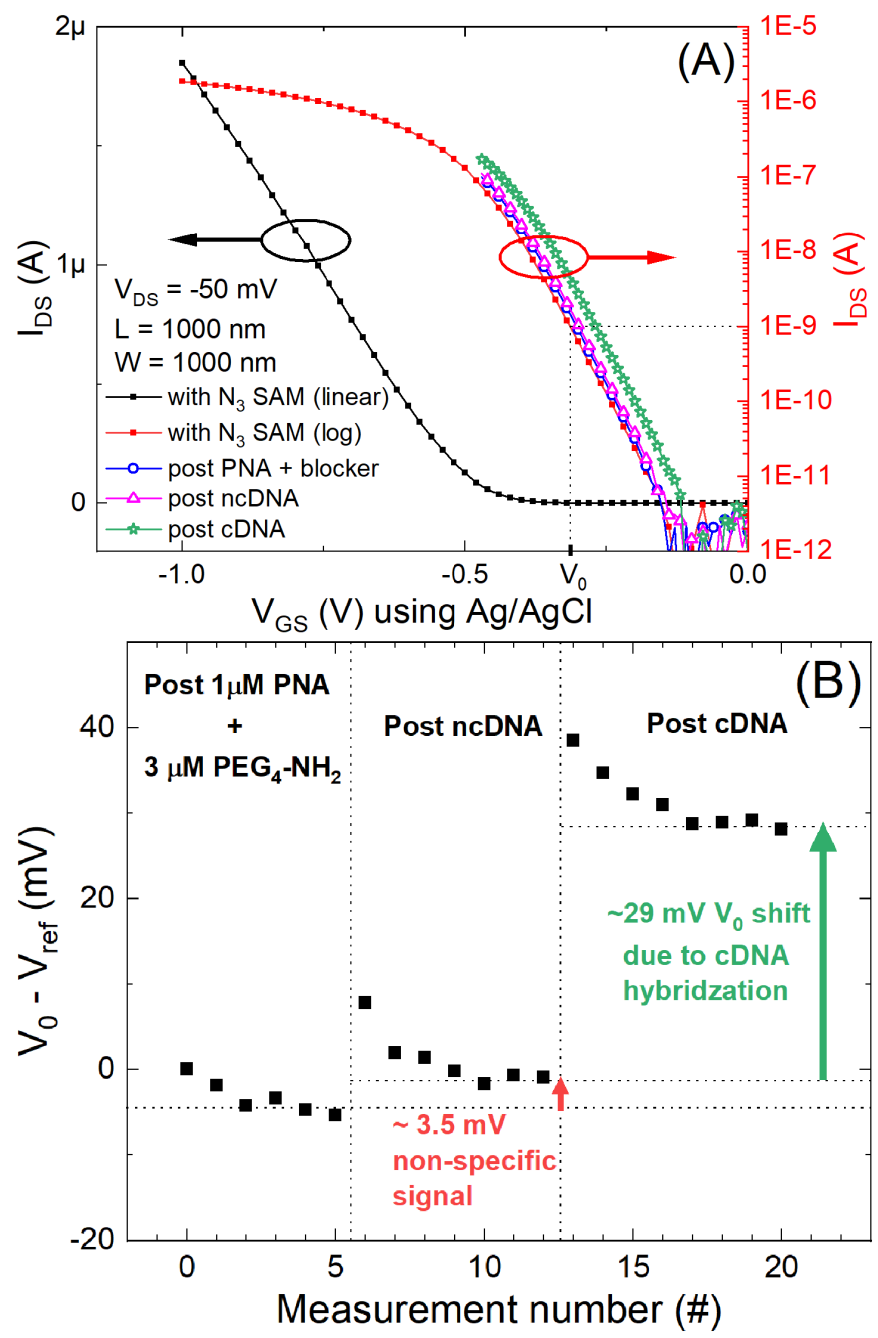

Fig. 5. (A) Transfer characteristics of a $1000 \mathrm{~nm}$ long and $1000 \mathrm{~nm}$ wide FET (at $\mathrm{V}_{\mathrm{DS}}=-50 \mathrm{mV}$ ) coated with $\mathrm{N}_{3}$-silane $\mathrm{SAM}$, measured in $1.5 \mathrm{mM}$ PBS buffer ( $\mathrm{pH} 7.2$ ) using a flow-through $\mathrm{Ag} / \mathrm{AgCl}$ as gate electrode. The plot also shows transfer characteristics after $(1 \mu \mathrm{M}$ PNA $+3 \mu \mathrm{M}$ positive blocker) grafting, ncDNA and cDNA incubation phases (also measured in 1.5 PBS buffer). The transfer curve shifts were quantified using constant current method, where a voltage $V_{0}$ (dotted vertical line) was defined as the gate voltage $V_{G S}$ for which the drain-source current $\mathrm{I}_{\mathrm{DS}}=1 \mathrm{nA}$ (dotted horizontal line). The FET gate voltage was swept down from $0 \mathrm{~V}$ until $\mathrm{I}_{\mathrm{DS}}$ exceeded $1 \mathrm{nA}$ (e.g. $\mathrm{V}_{\mathrm{GS}}=-0.5 \mathrm{~V}$ in this case) and the corresponding $V_{0}$ value (i.e. $V_{G S}$ at $I_{D S}=1 \mathrm{nA}$ ) was extracted for each measurement and for each incubation phase. (B) Post PNA/DNA incubation steps (in $150 \mathrm{mM}$ PBS) and a thorough rinse (in $1.5 \mathrm{mM}$ PBS)), multiple rinsing steps followed by I-V measurements were done (both in 1.5 mM PBS) until stable transfer curves was obtained. The plot shows the extracted $\left(\mathrm{V}_{0}-\mathrm{V}_{\text {ref }}\right)$ from each I-V measurement after PNA - blocker co-deposition and ncDNA and cDNA incubation steps, where $\mathrm{V}_{\text {ref }}=\mathrm{V}_{0}$ for measurement \#0.

$\mathrm{V}_{0}\left(\mathrm{~V}_{\mathrm{GS}}\right.$ for $\left.\mathrm{I}_{\mathrm{DS}}=1 \mathrm{nA}\right)$ values were extracted for each transfer curve and for each incubation step. These are shown in Figure 5 (B). Since the PNA and DNA incubations were done in $150 \mathrm{mM}$ PBS buffer and the electrical measurement in $1.5 \mathrm{mM}$ PBS (Table I), we made sure that complete buffer exchange took place by performing multiple rinsing steps followed by an I-V measurement (numbered successively in Figure 5 (B)) until stable transfer curves were obtained. The transfer curve after ncDNA incubation in Figure 5 (A) (for which the corresponding $\mathrm{V}_{0}$ is shown as measurement \#12 in Figure 5 (B)) almost overlaps with the transfer curve after 


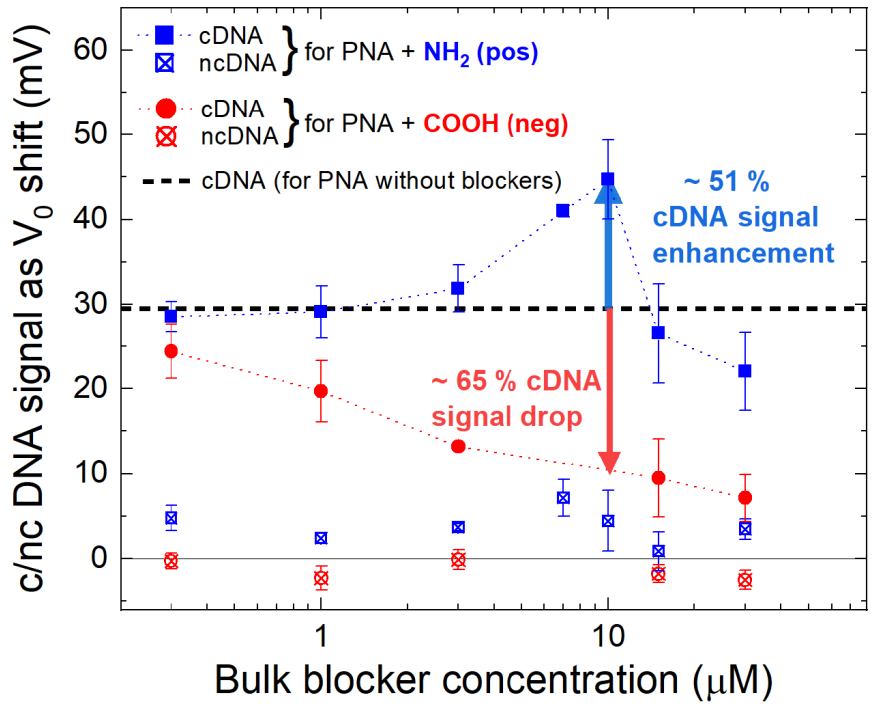

Fig. 6. FET signal ( $V_{0}$ shifts) for cDNA hybridization and for ncDNA as negative control, versus bulk blocker concentration (co-deposited with $1 \mu \mathrm{M}$ PNA) for both positive $\left(\mathrm{NH}_{2}\right)$ and negative $(\mathrm{COOH})$ blockers. The error bars are the standard deviations over 4 device measurements.

PNA-blocker co-deposition (measurement \#5, Figure 5 (B)), while after cDNA hybridization we observe a rigid shift in the transfer curve as shown in Figure 5 (A) (corresponding to measurement \#20, Figure 5 (B)). For the particular device shown in Figure 5, with $3 \mu \mathrm{M}$ positive $\left(-\mathrm{NH}_{2}\right)$ blocker co-deposited with $1 \mu \mathrm{M}$ PNA, we observed a negligible shift in $\mathrm{V}_{0}$ of $\sim 3.5 \mathrm{mV}$ for ncDNA incubation and about $29 \mathrm{mV}$ rigid $\mathrm{V}_{0}$ shift after cDNA hybridization.

The influence of changes in surface charge on the FET signal is shown in Figure 6. With increase in positive blocker concentration, we observed an increase in cDNA signal peaking at about $10 \mu \mathrm{M}$, and then a decrease in signal for higher blocker concentrations (Figure 6). The cDNA signal $\left(\mathrm{V}_{0}\right.$ shift $)$ at the peak is about $51 \%$ enhanced $(\sim 44 \mathrm{mV})$ compared to the cDNA signal without blocker molecules $(\sim 29 \mathrm{mV})$. When a negative blocker concentration was used, the $\mathrm{V}_{0}$ shift was not only less compared to the $\mathrm{V}_{0}$ shift for each corresponding positive blocker concentration but the $\mathrm{V}_{0}$ shift also continuously decreased with the increase in negative blocker concentration.

The peak observed in the FET signal (51\%) versus positive blocker concentration is much larger than the maximum increase in the bound DNA as measured by the Biacore (11\% for positive blocker and $16 \%$ for the negative blocker) and occurs at a different blocker concentration $(10 \mu \mathrm{M}$ vs. $3 \mu \mathrm{M})$. Moreover, the FET signal shows a peak only for positive surface modification and decreases monotonously for negative surface modification. By contrast, the binding measurements using Biacore show a nearly identical trend for both positive and negative blockers (Figure 3 (B)). This confirms that the dominant effect causing the peak in the FET signal is related to the charge of the blockers and the effect of screening (on FET signal transduction), and not a simple modulation of the amount of bound DNA.

With increase in positive blocker density on an initially negatively charged surface, the absolute potential drop over the EDL starts to decrease. This causes a decrease in overall ion concentration in the EDL until it becomes comparable to the bulk ion concentration. At that point the excess screening, caused by the strong, non-linear build-up of local ion concentration, vanishes, and the FET signal transduction is maximized. When the positive blocker concentration increases further, an oppositely charged EDL forms, and the screening increases again. Together with the gradual decrease in the amount of hybridized DNA, this results in a strong decrease in signal beyond the peak. For negative blockers, the absolute potential drop over the EDL keeps increasing, causing an increase in the total ion concentration in the EDL and a continuous increase in electrolytic screening of the hybridized cDNA, resulting in a continuous drop in FET signal transduction.

\section{Zeta Potential Measurements for Confirmation of Non-Linear Screening}

To further confirm the origin of the FET signal enhancement, we measured the zeta potentials before and after cDNA hybridization for a surface with a full layer of only PNA molecules (no blockers) and for a surface with $10 \mu \mathrm{M}$ positive blocker $+1 \mu \mathrm{M}$ PNA concentration (i.e. at the peak of the FET signal) (Figure 7 (A)). At the peak FET signal, the zeta potential swings almost symmetrically around zero, with a total swing of $105 \mathrm{mV}$. The initial surface charge is opposite to the analyte (DNA) charge but smaller, such that the net charge seen by the FET varies roughly between minus and plus half of the analyte charge. By contrast, for the PNA-only capture surface, the initial zeta potential is close to zero, such that the net charge seen by the FET varies between roughly zero and full analyte charge. Although there is a significant difference between the initial zeta potentials, the final zeta potentials seem to saturate, and the swing is reduced to $60 \mathrm{mV}$.

This can be understood qualitatively by considering the simplified case of a biomolecular layer with zero thickness (i.e. all the charges sit on the surface, and the slip plane coincides with the surface) and negligible charge inside the semiconductor (i.e. the EDL charge is exactly opposite to the surface charge). In this situation, the surface potential (which now coincides with the zeta potential) as a function of the surface charge is given by the Grahame equation [24] as shown in Figure 7 (B). We see that the maximum rate of change in zeta (or surface) potential occurs within a limited window around zero. When the zeta potential exceeds a few times $k_{B} T / q=25 \mathrm{mV}$, the sensitivity of the zeta potential to further changes in the charge decreases (the Debye-Hückel model based on a linearized Poisson-Boltzmann theory breaks down), giving rise to the excess non-linear screening. For a biomolecular layer of finite thickness, the analysis is more complex [4], [5] but the trend for the excess non-linear screening remains the same.

\section{E. Discussion and Outlook}

Here we experimentally investigated the impact of surface charge on the sensitivity of FETs to a large biomolecular density. In such a case, the analyte charge itself contributes to the 

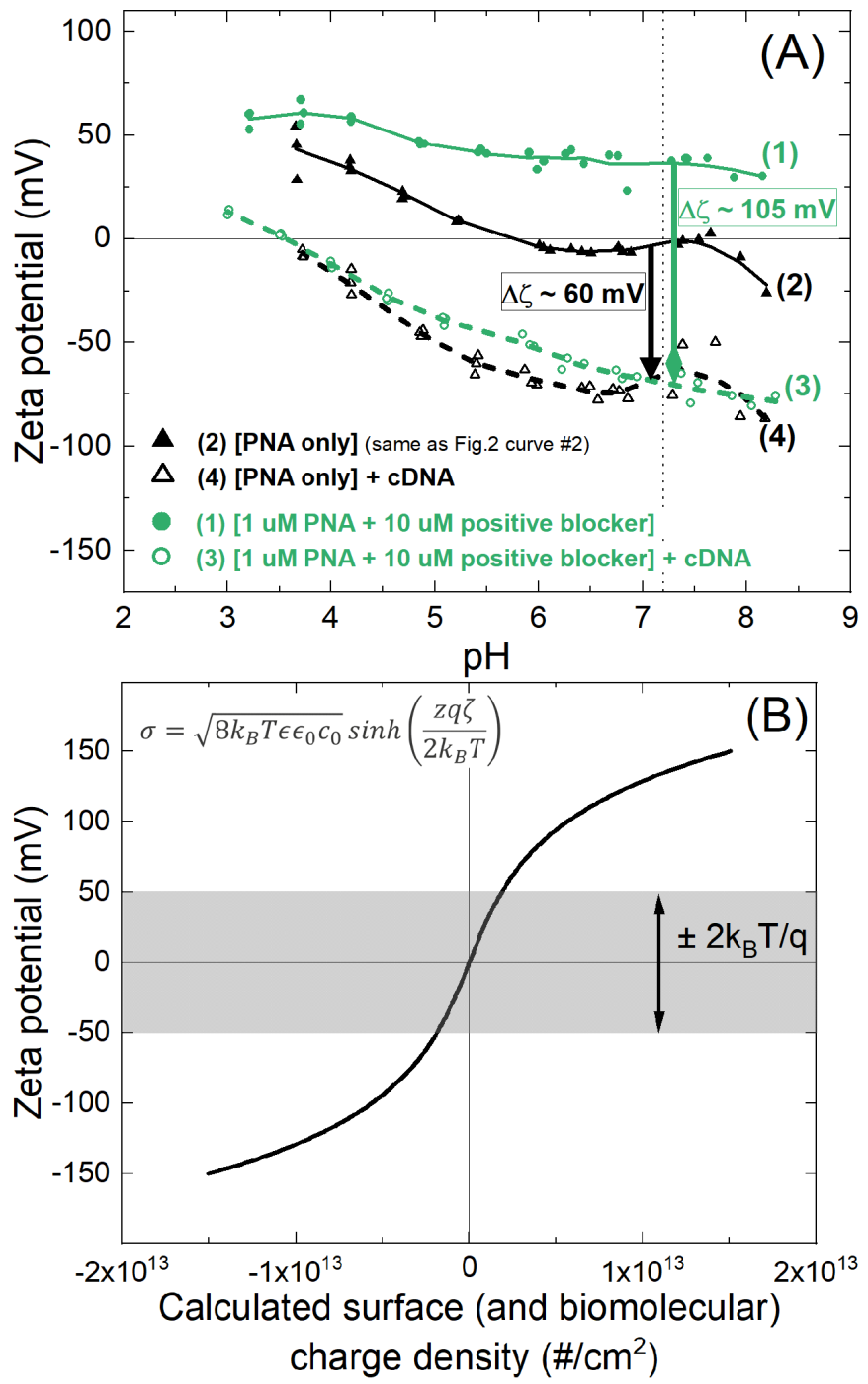

Fig. 7. (A) Zeta potential versus $\mathrm{pH}$ of the electrolyte (with dotted vertical line at $\mathrm{pH}$ 7.2), before and after cDNA hybridization for two different surface conditions: PNA only (curves \#2 and \#4) and $1 \mu \mathrm{M}$ PNA +10 $\mu \mathrm{M}$ positive blocker (curves \#1 and \#3). The black solid (\#2) and dashed curve (\#4) show surface after saturated PNA-only immobilization and cDNA hybridization respectively, giving a shift of about $60 \mathrm{mV}$ (black arrow with triangle head). Similarly, the green solid (\#1) and dashed curve (\#3) show surface after a mixed deposition of $1 \mu \mathrm{M}$ PNA + $10 \mu \mathrm{M}$ positive blocker and cDNA hybridization respectively, giving a larger shift of about $105 \mathrm{mV}$ (green arrow with rhombus head). (B) Plot for zeta potential $(\zeta)$ versus calculated surface (and biomolcular) charge density $(\sigma)$ using the Grahame equation (inset), where $\sigma$ is the calculated areal charge density at oxide-electrolyte interface, $c_{0}$ is the bulk ionic strength, $\epsilon$ and $\epsilon_{0}$ are absolute and relative permittivity constants, and $z$ is the valency of the bulk electrolyte $(z=1$ for $\mathrm{NaCl})$.

creation of a strong EDL, which gives rise to the excess nonlinear screening. Therefore, maximum sensitivity is obtained when the initial sensor surface is charged approximately half and opposite in polarity, of the expected biomolecular charge, such that the EDL potential swings roughly symmetrically around zero. In case of a small biomolecular density or even single molecule sensing however, the analyte itself does not significantly influences the EDL, and the sensor surface potential should be close to zero $\mathrm{mV}$ (i.e. an uncharged surface), allowing the ion concentration in the EDL to be the same as the bulk ionic strength.
One last point merits further discussion. The change in the zeta potential in response to the DNA hybridization is significantly larger than the signal picked up by the FET, both at the peak $(105 \mathrm{mV}$ vs $44 \mathrm{mV})$ and on the pure PNA capture probes without additional surface charge control $(60 \mathrm{mV}$ vs $29 \mathrm{mV}$ ). As the FET measures the surface potential $\Psi_{0}$, this points towards an additional negative feedback (or screening) mechanism that suppresses the change in surface potential compared to the changes at the slip plane deeper inside the liquid. This mechanism has also been identified in some early works [4]-[7], but has been largely ignored since then. It is likely related to screening by the $\mathrm{pH}$-sensitive surface states.

Our experiments and the discussion so far have focused on the role of the electrolyte screening i.e. the accumulation and depletion of mobile electrolyte ions when the arrival of an analyte charge (tries to) modify the local potential. This accumulation/depletion forms a negative feedback loop that opposes (or suppresses) the change in potential. We have studied the non-linear increase in electrolyte screening when a large potential drop is present in the EDL in response to a large density of surface charge (and/or functional layer and analyte charges). Up till now we have implicitly assumed that these charges are fixed, i.e. that they don't change in response to the change in local potential.

However, this is not true for the silanol ( $\mathrm{Si}-\mathrm{OH})$ groups on the $\mathrm{SiO}_{2}$ surface. Their protonation (or charge state) is in equilibrium with the surface $\mathrm{pH}$, and the surface $\mathrm{pH}$ varies with the surface potential $\Psi_{0}$ through the Boltzmann relation, $a_{H_{s}^{+}}=a_{H_{b}^{+}} \exp \left(-q \Psi_{0} / k_{B} T\right)$, with $a_{H_{s}^{+}}$and $a_{H_{b}^{+}}$the surface and bulk proton activities [25], [26]. Similar to the mobile electrolyte ions, when the arrival of an analyte charge (tries to) modify the surface potential, the charge in the surface states will change, forming a negative feedback loop that opposes (or suppresses) the change in potential. This results in screening of the analyte charge by the $p H$-sensitive surface states. The resulting tendency to pin the surface potential by the pH-sensitive surface groups is clearly visible in e.g. Fig. 3 of Landheer et al. [4], where the potential profiles without and with the analyte charge diverge from the bulk solution towards the outer edge of the analyte layer (approximate position of the slip plane where our zeta potentials are measured), and converge again towards the surface (where our FET signal is obtained) when $\mathrm{pH}$ sensitive surface states are present. This pinning effect is strongest for surfaces with the highest $\mathrm{pH}$ sensitivity, i.e. surfaces where the surface potential is tied the strongest to the $\mathrm{pH}$ of the bulk solution [4]-[7]. Hence, as already concluded by Wunderlich et al. [6] "a quantitative detection of charged molecules by FETs is best achieved on surfaces with both low $\mathrm{pH}$ sensitivity and low surface potential". The negative correlation between $\mathrm{pH}$ sensitivity and bio-sensitivity has recently been observed experimentally [27].

Hence, in future work, it will be important to consider both, the sensor surface potential (or charge) and the $\mathrm{pH}$ sensitivity at the same time, such that the optimization for high FET sensitivity is implemented not only by reducing the absolute surface charge but also by minimizing $\mathrm{pH}$ sensitivity, by either selecting appropriate surfaces with low intrinsic pH sensitivity or by using dedicated surface chemistries to 
passivate or compensate the effect of the surface (silanol) groups.

\section{CONCLUSION}

We experimentally investigated the impact of excess nonlinear electrolytic screening on the sensitivity of bioFETs, when the local potential within the EDL exceeds a few times $k_{B} T / q=25 \mathrm{mV}$, and the linear Debye-Hückel screening model is no longer accurate. We used variable concentrations of either positively or negatively charged blocker molecules, co-deposited with the PNA capture probes, to modulate the overall surface charge of $\mathrm{SiO}_{2}$-based FET and demonstrated an improvement in FET sensitivity to hybridized cDNA when the net charge is minimized.

Reference measurements using surface plasmon resonance showed only minor changes in the amount of cDNA hybridization (11 to $16 \%$ ), related to small changes in steric effect and capture probe density, and identical for positive and negative surface modifications. The electrical FET measurements gave a clear difference in response between positive and negative surface modifications. Positive blockers, with their opposite charge to $\mathrm{SiO}_{2}$ surface and DNA analyte (both negative), resulted in an increase of up to $51 \%$ in the FET signal, followed by a decrease when the positive blocker concentration was further increased. Negative surface modification on the other hand resulted in a monotonic decrease in FET sensitivity. Zeta potential measurements confirm that the peak in the FET sensitivity is obtained when the overall charge (or potential) in the EDL is close to zero. For small analyte charge, e.g. in single molecule measurements, the maximum signal would be obtained when the net charge in the double layer is zero. This is in agreement with earlier theoretical predictions [4]-[6] and can be understood as excess or non-linear electrolyte screening when the linear Debye-Hückel model breaks down, i.e. when the potential in the double layer exceeds a few times $k_{B} T / q=25 \mathrm{mV}$. In such a situation, electrolyte screening is no longer related to the bulk ionic strength only, but the local ionic concentrations in the double layer need to be taken into account as well.

We further found that the bioFET signal (the change in surface potential) is smaller than the change in zeta potential, which may be a signature of additional screening by the $\mathrm{pH}$-sensitive surface states that was predicted in the same theoretical studies and observed experimentally by Kumar et al. [27]. Surface treatments to maximize the bioFET response should aim at minimizing both the double layer potential and the $\mathrm{pH}$ sensitivity of the sensor.

\section{ACKNOWLEDGMENT}

The authors would like to thank Bert Du Bois and Simone Severi for the FinFET fabrication process development, Dino Ruic for useful discussions, Rita Vos and Karolien Jans for discussions on surface chemistry and zeta potential measurements, Kris Covens and Tim Steylaerts for Biacore training and sample preparation, Kim Baumans, Rudy Verheyen and Erwin Vandenplas for their help in microfabrication deposition processes, and Maria Vidal Cabanilles for help in surface functionalization.

\section{References}

[1] B. M. Lowe, K. Sun, I. Zeimpekis, C.-K. Skylaris, and N. G. Green, "Field-effect sensors-from $\mathrm{pH}$ sensing to biosensing: Sensitivity enhancement using streptavidin-biotin as a model system," Analyst, vol. 142, no. 22, pp. 4173-4200, 2017.

[2] F. N. Ishikawa et al., "A calibration method for nanowire biosensors to suppress device-to-device variation," ACS Nano, vol. 3, no. 12, pp. 3969-3976, Dec. 2009.

[3] J. Fritz, E. B. Cooper, S. Gaudet, P. K. Sorger, and S. R. Manalis, "Electronic detection of DNA by its intrinsic molecular charge," Proc. Nat. Acad. Sci. USA, vol. 99, no. 22, pp. 14142-14146, Oct. 2002.

[4] D. Landheer, G. Aers, W. R. McKinnon, M. J. Deen, and J. C. Ranuarez, "Model for the field effect from layers of biological macromolecules on the gates of metal-oxide-semiconductor transistors," J. Appl. Phys., vol. 98, no. 4, Aug. 2005, Art. no. 044701.

[5] D. Landheer, W. R. McKinnon, G. Aers, W. Jiang, M. J. Deen, and M. W. Shinwari, "Calculation of the response of field-effect transistors to charged biological molecules," IEEE Sensors J., vol. 7, no. 9, pp. 1233-1241, Sep. 2007.

[6] B. K. Wunderlich, P. A. Neff, and A. R. Bausch, "Mechanism and sensitivity of the intrinsic charge detection of biomolecular interactions by field effect devices," Appl. Phys. Lett., vol. 91, no. 8, pp. 1-4, 2007.

[7] D. Landheer, W. R. McKinnon, W. H. Jiang, and G. Aers, "Effect of screening on the sensitivity of field-effect devices used to detect oligonucleotides," Appl. Phys. Lett., vol. 92, no. 25, pp. 2006-2009, 2008.

[8] S. Ingebrandt and A. Offenhäusser, "Label-free detection of DNA using field-effect transistors," Phys. Status Solidi A, vol. 203, no. 14, pp. 3399-3411, Nov. 2006.

[9] Y. Liu and R. W. Dutton, "Effects of charge screening and surface properties on signal transduction in field effect nanowire biosensors," J. Appl. Phys., vol. 106, no. 1, Jul. 2009, Art. no. 014701.

[10] K. Shoorideh and C. O. Chui, "Optimization of the sensitivity of FETbased biosensors via biasing and surface charge engineering," IEEE Trans. Electron Devices, vol. 59, no. 11, pp. 3104-3110, Nov. 2012.

[11] K. Jayant, K. Auluck, S. Rodriguez, Y. Cao, and E. C. Kan, "Programmable ion-sensitive transistor interfaces. III. Design considerations, signal generation, and sensitivity enhancement," Phys. Rev. E, Stat. Phys. Plasmas Fluids Relat. Interdiscip. Top., vol. 89, no. 5, pp. 1-12, May 2014.

[12] K. Martens et al., "BioFET technology: Aggressively scaled pMOS FinFET as biosensor," in IEDM Tech. Dig., Dec. 2019, pp. 438-441.

[13] M. Gupta et al., "Size independent sensitivity to biomolecular surface density using nanoscale CMOS technology transistors," IEEE Sensors J., vol. 20, no. 16, pp. 8956-8964, Aug. 2020, doi: 10.1109/jsen.2020. 2987627.

[14] E. Altamirano-Sánchez, T. Vandeweyer, and W. Boullart, "Dry etching patterning requirements for multi-gate devices," in CMOS Nanoelectronics: Innovative Devices, Architectures, and Applications, N. Collaert, Ed. Singapore: Pan Stanford Publishing Pte. Ltd, 2012, pp. 29-55.

[15] A. Veloso et al., "Gate-all-around NWFETs vs. triple-gate FinFETs: Junctionless vs. Extensionless and conventional junction devices with controlled EWF modulation for multi-VT CMOS," in Proc. Symp. VLSI Technol. (VLSI Technol.), Jun. 2015, pp. T138-T139.

[16] R. Vos et al., "Chemical vapor deposition of azidoalkylsilane monolayer films," Langmuir, vol. 34, no. 4, pp. 1400-1409, Jan. 2018.

[17] J. Ryken et al., "Biosensing with $\mathrm{SiO}_{2}$-covered SPR substrates in a commercial SPR-tool," Sens. Actuators B, Chem., vol. 200, pp. 167-172, Sep. 2014.

[18] M. Gupta et al., "Size independent $\mathrm{pH}$ sensitivity for ion sensitive FinFETs down to $10 \mathrm{~nm}$ width," IEEE Sensors J., vol. 19, no. 16, pp. 6578-6586, Aug. 2019.

[19] J. M. Berg, J. L. Tymoczko, and L. Stryer, Biochemistry, 5th ed. New York, NY, USA: W. H. Freeman, 2002.

[20] U. Jonsson et al., "Real-time biospecific interaction analysis using surface plasmon resonance and a sensor chip technology," Biotechniques, vol. 11, no. 5, pp. 620-627, 1991.

[21] E. Stenberg, B. Persson, H. Roos, and C. Urbaniczky, "Quantitative determination of surface concentration of protein with surface plasmon resonance using radiolabeled proteins," J. Colloid Interface Sci., vol. 143, no. 2, pp. 513-526, May 1991.

[22] P. Gong and R. Levicky, "DNA surface hybridization regimes," Proc. Nat. Acad. Sci. USA, vol. 105, no. 14, pp. 5301-5306, Apr. 2008.

[23] A. W. Peterson, "The effect of surface probe density on DNA hybridization," Nucleic Acids Res., vol. 29, no. 24, pp. 5163-5168, Dec. 2001. 
[24] H.-J. Butt, K. Graf, and M. Kappl, "The electric double layer," in Physics and Chemistry of Interfaces. Weinheim, Germany: Wiley-VCH Verlag GmbH Co. KGaA, 2004, pp. 42-56.

[25] L. Bousse, N. F. De Rooij, and P. Bergveld, "Operation of chemically sensitive field-effect sensors as a function of the insulator-electrolyte interface," IEEE Trans. Electron Devices, vol. 30, no. 10, pp. 1263-1270, Oct. 1983.

[26] R. E. G. van Hal, J. C. T. Eijkel, and P. Bergveld, "A general model to describe the electrostatic potential at electrolyte oxide interfaces," Adv. Colloid Interface Sci., vol. 69, nos. 1-3, pp. 31-62, Dec. 1996.

[27] N. Kumar, S. Kumar, J. Kumar, and S. Panda, "Investigation of mechanisms involved in the enhanced label free detection of prostate cancer biomarkers using field effect devices," J. Electrochem. Soc., vol. 164 no. 9, pp. B409-B416, 2017.

Mihir Gupta (Member, IEEE) received the B.Tech. degree in electronics and communication engineering from Rajasthan Technical University, India, in 2012, and the joint M.Sc. degree in microelectronics from Nanyang Technological University, Singapore, and the Technische Universität München, Germany, in 2015. He is currently pursuing the Ph.D. degree with the Department of Physics and Astronomy, Katholieke Universiteit (KU) Leuven, in collaboration with imec (life science technologies) in Belgium. His research interest includes sensor development for medical or chemical applications using semiconductor technology and advanced lithography.

Sybren Santermans (Member, IEEE) received the B.S. degree in mechanical engineering and the M.S. degree in nanoscience, nanotechnology, and nanoengineering from Katholieke Universiteit (KU) Leuven, Leuven, Belgium, in 2015 and 2017, respectively. He is currently pursuing the Ph.D. degree in materials engineering with $\mathrm{KU}$ Leuven in collaboration with imec, Leuven. His research interests include the characterization, modeling, and the simulation of bioFET sensors.

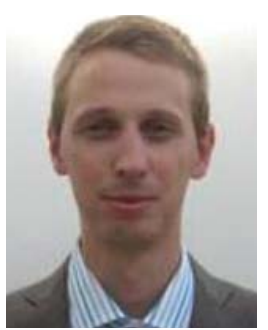

Geert Hellings (Senior Member, IEEE) received the B.S. and M.S. degrees in electrical engineering from Katholieke Universiteit (KU) Leuven, Belgium, in 2007, and the Ph.D. degree from the Electrical Engineering Department (ESAT), Integrated Systems Division (INSYS), KU Leuven, and the CMOS Technology Department, imec, Belgium, in 2012. The main topic of his work was the integration of advanced field effect transistors with high-mobility channel materials and heterostructure confinement for digital logic applications. In 2012, he joined the Device Reliability and Electrical Characterization Group, imec, researching electrostatic discharge events in integrated circuitry. He holds several patents and has authored or coauthored approximately 170 technical articles for publication in journals and presentations at conferences. He won the IEEE Region Eight Student Paper Contest in 2008 and received the imec Scientific Excellence Award in 2011.
Liesbet Lagae received the Ph.D. degree from Katholieke Universiteit (KU) Leuven, Belgium, for her work on magnetic random access memories obtained under an IWT Grant. She is the Co-Founder and currently the Program Director of Life Science Technologies with imec. In this role, she oversees the emerging Research and Development, the public funded activities and early business creation. As a young group leader, she has initiated the field of molecular and cellular biochips leveraging silicon technologies at imec, Belgium. The life science program has grown from emerging activities to a mature business line that provides smart silicon chip solutions to the life science industry. The applications include medical diagnostics, point-of-care solutions, DNA sequencing, cytometry, bioreactors, neuroprobes, and implants. She holds a prestigious ERC consolidator grant for developing a platform on single cell analysis and sorting. She has coauthored 125 peer-reviewed articles in international journals and holds 15 patents in the field. She is also a parttime Professor of Nanobiotechnology with the Department of Physics, KU Leuven.

Koen Martens (Member, IEEE) received the Ph.D. degree in electrical engineering from Katholieke Universiteit (KU) Leuven, Belgium, in 2009. $\mathrm{He}$ is currently a Research Scientist with imec, Belgium. He has worked on germanium and III-V MOSFET technology with imec and Stanford University in 2006. He has done exploratory nanoelectronics work on the metal-insulator transition material VO2 with imec and IBM in 2012. He was actively involved in the EU 2D Nanolattice MOSFET Project and STT MRAM Program at imec. His current research interests at imec and KU Leuven include ISFETs and bioFETs, respectively, and the voltage control of magnetic anisotropy (VCMA).

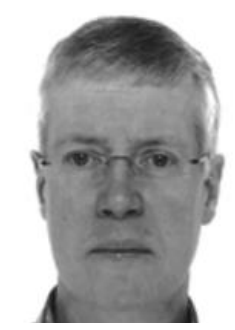

Willem Van Roy received the master's and $\mathrm{Ph} . \mathrm{D}$. degrees in electrical engineering from Katholieke Universiteit (KU) Leuven, Leuven, Belgium, in 1990 and 1995, respectively.

He joined imec, Leuven, in 1990. From 1995 to 1999, he was a Postdoctoral Researcher at the Joint Research Center for Atom Technology (JRCAT), Tsukuba, Japan, before returning to imec. He was active for about 20 years in the fields of magnetoelectronics and spintronics, focusing on the materials science and device physics of spins in semiconductors and metallic heterostructures. Since 2008, his interest has been shifted to the life sciences area, and he is currently a Principal Member of Technical Staff of the Department of Life Science Technologies, imec, focusing on optical and electrical biosensing, overall system performance analysis, assay kinetics, with further interests in ionics, microfluidics, PCR, electrochemistry, and more. $\mathrm{He}$ has authored or coauthored over 140 peer-reviewed journal articles and holds 15 patent applications. 